\title{
ON A PROBLEM OF CHEN AND FANG RELATED TO INFINITE ADDITIVE COMPLEMENTS
}

\author{
SÁNDOR Z. KISS AND CSABA SÁNDOR
}

\begin{abstract}
Two infinite sets $A$ and $B$ of nonnegative integers are called additive complements if their sumset contains every nonnegative integer. In 1964, Danzer constructed infinite additive complements $A$ and $B$ with $A(x) B(x)=(1+o(1)) x$ as $x \rightarrow \infty$, where $A(x)$ and $B(x)$ denote the counting function of the sets $A$ and $B$, respectively. In this paper we solve a problem of Chen and Fang by extending the construction of Danzer.
\end{abstract}

\section{INTRODUCTION}

Let $\mathbb{N}$ be the set of nonnegative integers and let $A$ and $B$ be infinite sets of nonnegative integers. We define their sum by $A+B=\{a+b: a \in A, b \in B\}$. We say $A$ and $B$ are infinite additive complements if their sum contains all nonnegative integers i.e., $A+B=\mathbb{N}$. Let $A(x)$ be the number of elements of $A$ up to $x$ i.e.,

$$
A(x)=\sum_{\substack{a \in A \\ a \leq x}} 1 .
$$

Since $A$ and $B$ are infinite additive complements, every nonnegative integer $x$ can be written in the form $a+b=x$, where $a \in A, b \in B$. Then clearly [7] we have $A(x) B(x) \geq x+1$, which implies that

$$
\limsup _{x \rightarrow \infty} \frac{A(x) B(x)}{x} \geq \liminf _{x \rightarrow \infty} \frac{A(x) B(x)}{x} \geq 1
$$

According to a conjecture of H. Hanani [3], the above result can be sharpened in the following way.

Conjecture 1.1 (Hanani, 1957). If $A$ and $B$ are infinite additive complements, then

$$
\limsup _{x \rightarrow \infty} \frac{A(x) B(x)}{x}>1
$$

Later, Danzer [2] disproved the above conjecture of Hanani.

2020 Mathematics Subject Classification. Primary 11B13; Secondary 11B34.

Key words and phrases. additive number theory, additive complement counting function, sumset. 
Theorem 1.2 (Danzer, 1964). There exist infinite additive complements $A$ and $B$ such that

$$
\lim _{x \rightarrow \infty} \frac{A(x) B(x)}{x}=1 .
$$

Let $A_{1}, \ldots, A_{r}$ be infinite sets of nonnegative integers. We define their sum by $A_{1}+A_{2}+\ldots+A_{r}=\left\{a_{1}+a_{2}+\ldots+a_{r}: a_{i} \in A_{i}, 1 \leq i \leq r\right\}$. Chen and Fang extended the notion of additive complements to more than two sets in the following way [1]. The infinite sets $A_{1}, \ldots, A_{r}$ of nonnegative integers are said to form infinite additive complements if their sum contains all nonnegative integers. Again, it is easy to see that $A_{1}(x) \cdots A_{r}(x) \geq$ $\left(A_{1}+\ldots+A_{r}\right)(x)=x+1$, thus

$$
\liminf _{x \rightarrow \infty} \frac{A_{1}(x) \cdots A_{r}(x)}{x} \geq 1 .
$$

Furthermore, they posed the following problem.

Problem 1.3. For each integer $r \geq 3$ find additive complements $A_{1}, \ldots, A_{r}$ such that

$$
\lim _{x \rightarrow \infty} \frac{A_{1}(x) \cdots A_{r}(x)}{x}=1
$$

In this paper we solve this problem. Note that our construction is the extension of Danzer's result to $r>2$.

Theorem 1.4. For each integer $h \geq 2$ there exist infinite sets of nonnegative integers $A_{1}, \ldots, A_{h}$ with the following properties:

(1) $A_{1}+\ldots+A_{h}=\mathbb{N}$,

(2) $A_{1}(x) \cdots A_{h}(x)=(1+o(1)) x$ as $x \rightarrow \infty$.

Let $R_{A+B}(n)$ be the number of representations of the integer $n$ in the form $a+b=n$, where $a \in A, b \in B$. W. Narkiewicz [4] proved the following theorem.

Theorem 1.5 (Narkiewicz, 1960). If $R_{A+B}(n) \geq C$ for every sufficiently large integer $n$, where $C$ is a constant and

$$
\limsup _{x \rightarrow \infty} \frac{A(x) B(x)}{x} \leq C,
$$

then

$$
\lim _{x \rightarrow \infty} \frac{A(2 x)}{x}=1,
$$

or

$$
\lim _{x \rightarrow \infty} \frac{B(2 x)}{x}=1 .
$$


Additive complements $A, B$ are called exact if $A(x) B(x)=(1+o(1)) x$ as $x \rightarrow \infty$. For any $h \geq 2$ integer let us define the system of sets $\mathcal{A}_{h}$ by

$$
\begin{gathered}
\mathcal{A}_{h}=\left\{A \subset \mathbb{N}: \text { there exist } A_{2}, \ldots, A_{h} \subset \mathbb{N},\right. \\
\left.A+A_{2}+\ldots+A_{h}=\mathbb{N}, A(x) \cdot A_{2}(x) \cdots A_{h}(x)=(1+o(1)) x \text { as } x \rightarrow \infty\right\} .
\end{gathered}
$$

Theorem 1.4 implies that $\mathcal{A}_{h} \neq \emptyset$ for every $h \geq 2$. We prove that the $\mathcal{A}_{h}$ 's form an infinite chain.

Theorem 1.6. We have $\mathcal{A}_{2} \supseteq \mathcal{A}_{3} \supseteq \ldots$

It follows from Theorem 1.6 that if $A \in \mathcal{A}_{2}$, then $A(x)=x^{o(1)}$ or $A(x)=$ $x^{1+o(1)}$ as $x \rightarrow \infty$. Then for any $h \geq 2, A \in \mathcal{A}_{h}$ implies that $A(x)=x^{o(1)}$ or $A(x)=x^{1+o(1)}$ as $x \rightarrow \infty$. If the sets $A_{1}, \ldots, A_{h} \subset \mathbb{N}$ satisfy $A_{1}+\ldots+A_{h}=$ $\mathbb{N}$ and $A_{1}(x) \cdots A_{h}(x)=(1+o(1)) x$ as $x \rightarrow \infty$, then $A_{i}(x)=x^{1+o(1)}$ or $A_{i}(x)=x^{o(1)}$ for every $1 \leq i \leq h$ while $x \rightarrow \infty$. As a corollary, one can get from Theorem 1.4 that

Corollary 1.7. Let $A_{1}, \ldots, A_{h}$ be infinite sets of nonnegative integers such that $A_{1}+\ldots+A_{h}=\mathbb{N}$ and

$$
A_{1}(x) \cdots A_{h}(x)=(1+o(1)) x
$$

as $x \rightarrow \infty$. Then there exists an index $i$ such that $A_{i}(x)=x^{1+o(1)}$ and $A_{j}(x)=x^{o(1)}$ for every $1 \leq j \leq h$ with $j \neq i$ as $x \rightarrow \infty$.

We pose the following problems for further research.

Problem 1.8. Does $\mathcal{A}_{h} \neq \mathcal{A}_{h+1}$ hold for every $h \geq 2$ ?

Problem 1.9. Assume that $A_{1}+\ldots+A_{h}=\mathbb{N}$ and $A_{1}(x) \cdots A_{h}(x)=$ $(1+o(1)) x$ hold as $x \rightarrow \infty$. Does there exist a permutation $i_{1}, \ldots, i_{h}$ of the indices $1, \ldots, h$ such that $A_{i_{j}}(x)=\left(A_{i_{j-1}}(x)\right)^{o(1)}$ for every $2 \leq j \leq h$ as $x \rightarrow \infty$ ?

The statement in Problem 1.9 holds for $h=2$.

The exact complemets have been investigated by many authors in the last few decades. In particular, they studied what kind of sets $A$ of nonnegative integers with $A(x)=x^{o(1)}$ as $x \rightarrow \infty$ have exact additive complement. It was proved in [2] that the sequence $a_{n}=(n !)^{2}+1$ has an exact complement. In [5] Ruzsa showed that the set of the powers of an integer $a \geq 3$ has an exact complement. Furthermore, in [6] he proved that the set of powers of 2 has an exact complement. Moreover, he also proved in [6] that $A=\left\{a_{1}, a_{2}, \ldots\right\}$ with $1 \leq a_{1}<a_{2}<\ldots$ has an exact complement if $\lim _{n \rightarrow \infty} \frac{a_{n+1}}{n a_{n}}=\infty$. In view of these results, it is natural to ask 
Problem 1.10. Is it true that if $A \in \mathcal{A}_{2}, A(x)=x^{o(1)}$ as $x \rightarrow \infty$, then $A(x)=O(\log x)$ ?

\section{Proof of TheOrem 1.4}

For any nonnegative integers $a<b$, let us define $[a, b]=\{x \in \mathbb{N}: a \leq$ $x \leq b\}$. The following lemma plays the key role in the proof of Theorem 1.4 .

Lemma 2.1. Assume that $A_{1}, \ldots, A_{h} \subset \mathbb{N}$ are infinite subsets with the following properties

(1) $A_{1}+\ldots+A_{h}=\mathbb{N}$

(2) there exists a monotone increasing arithmetic function $f_{h}(n) \geq 0$ with

$$
\lim _{n \rightarrow \infty} f_{h}(n)=\infty
$$

such that the equation $a_{1}+\ldots+a_{h}=n, a_{i} \in A_{i}$ has a solution with $a_{i} \geq f_{h}(n)$,

(3) $A_{1}(x) \cdots A_{h}(x)=(1+o(1)) x$ as $x \rightarrow \infty$.

For $m \in \mathbb{N}$, let $g(m)$ be an integral-valued strictly increasing function such that $g\left(f_{h}(n)\right) \geq n^{2}$ for every $n \in \mathbb{N}$. Put for shortness

$$
\begin{gathered}
\Phi_{n}=g(n+1) !+h(g(n+1)-1) !, \\
\Delta_{n}=n-\lceil\sqrt{n}\rceil,
\end{gathered}
$$

and for $n \geq 6$ let

$$
M_{n}=\left[g(n) !-2 \Delta_{n}, \Phi_{n}\right] .
$$

Furthermore, for $1 \leq i \leq h$, let $B_{i}=\{0\} \cup\left\{g(a) !+a: a \in A_{i}\right\}$ and define the sets of integers

$$
B_{h+1}=\left\{a: 0 \leq a \leq \Phi_{5}-1\right\} \cup \bigcup_{n \geq 6}\left\{\alpha \in M_{n}: \Delta_{n} \mid \alpha\right\} .
$$

Then

(i) $B_{1}+\ldots+B_{h+1}=\mathbb{N}$,

(ii) there exists a monotone increasing arithmetic function $f_{h+1}(n) \geq 0$ with

$$
\lim _{n \rightarrow \infty} f_{h+1}(n)=\infty
$$

such that the equation $b_{1}+\ldots+b_{h+1}=n, b_{i} \in B_{i}$ has a solution with $b_{i} \geq f_{h+1}(n)$,

(iii) $B_{1}(x) \cdots B_{h+1}(x)=(1+o(1)) x$ as $x \rightarrow \infty$. 
2.1. Proof of the lemma. Now we prove that for any $N \geq 6$,

$$
B_{1}+\ldots+B_{h}+\left\{\alpha \in M_{N}: \Delta_{N} \mid \alpha\right\} \supseteq\left[\Phi_{N-1}-2 \Delta_{N}+N, \Phi_{N}\right] .
$$

Consider an element from the interval on the right hand side i.e., let $y$ be

$$
\Phi_{N-1}-2 \Delta_{N}+N \leq y \leq \Phi_{N}
$$

It is clear that there exists an $\lceil\sqrt{N}\rceil \leq m \leq N-1$ with $y \equiv m\left(\bmod \Delta_{N}\right)$. By (2), there exist $a_{1}, \ldots, a_{h}$ integers with $a_{i} \in A_{i}$ such that $m=a_{1}+\ldots+a_{h}$ and $a_{i} \geq f_{h}(m)$. Since $f_{h}(m)$ is a monotone increasing function and $g(m)$ is a strictly increasing function, we have

$$
g\left(a_{i}\right) \geq g\left(f_{h}(m)\right) \geq g\left(f_{h}(\lceil\sqrt{N}\rceil)\right) \geq(\lceil\sqrt{N}\rceil)^{2} \geq N
$$

and so $g\left(a_{i}\right) ! \equiv 0\left(\bmod \Delta_{N}\right)$. Let $b_{i}=g\left(a_{i}\right) !+a_{i}$. Then $b_{i} \in B_{i}$ for every $1 \leq i \leq h$. It follows that

$$
\sum_{i=1}^{h} b_{i}=\sum_{i=1}^{h}\left(g\left(a_{i}\right) !+a_{i}\right) \equiv \sum_{i=1}^{h} a_{i} \equiv m \equiv y \quad\left(\bmod \Delta_{N}\right),
$$

which implies that $\frac{y-\left(b_{1}+\ldots+b_{h}\right)}{\Delta_{N}}$ is an integer and clearly

$$
y=b_{1}+\ldots+b_{h}+\frac{y-\left(b_{1}+\ldots+b_{h}\right)}{\Delta_{N}} \cdot \Delta_{N} .
$$

In view of these facts, it is enough to show that

$$
g(N) !-2 \Delta_{N} \leq y-\left(b_{1}+\ldots+b_{h}\right) \leq \Phi_{N} .
$$

Since $g(n)$ is a strictly increasing function, we have

$0 \leq b_{i}=g\left(a_{i}\right) !+a_{i} \leq g(m) !+m \leq g(N-1) !+N-1<(g(N)-1) !+N$

and so

$$
0 \leq \sum_{i=1}^{h} b_{i}<h((g(N)-1) !+N)
$$

It follows that

$$
\begin{gathered}
y-\left(b_{1}+\ldots+b_{h}\right) \geq y-h((g(N)-1) !+N) \\
\geq g(N) !-2(N-\lceil\sqrt{N}\rceil)+h((g(N)-1) !+N)-h(g(N)-1) !+N) \\
=g(N) !-2 \Delta_{N}
\end{gathered}
$$

and

$$
y-\left(b_{1}+\ldots+b_{h}\right) \leq y \leq \Phi_{N} .
$$

Thus for $N \geq 6$, we have

$$
B_{1}+\ldots+B_{h+1} \supseteq\left[\Phi_{N-1}-2 \Delta_{N}+N, \Phi_{N}\right] \supseteq\left[\Phi_{N-1}, \Phi_{N}\right] .
$$


This implies that

$$
B_{1}+\ldots+B_{h+1} \supseteq \bigcup_{N \geq 6}\left[\Phi_{N-1}, \Phi_{N}\right]=\left[\Phi_{5},+\infty\right) .
$$

Moreover, for $1 \leq i \leq h, 0 \in B_{i}$ and $B_{h+1} \supseteq\left[0, \Phi_{5}-1\right]$. Therefore,

$$
\left[0, \Phi_{5}-1\right] \subseteq B_{1}+\ldots+B_{h+1}
$$

and so $B_{1}+\ldots+B_{h+1}=\mathbb{N}$, which proves (i).

If $\Phi_{N-1} \leq n \leq \Phi_{N}$, then there exists a representation $n=b_{1}+\ldots+b_{h+1}$, where $b_{i}=g\left(a_{i}\right) !+a_{i} \geq a_{i} \geq f_{h}(\lceil\sqrt{N}\rceil)$ and $b_{h+1} \geq g(N) !-2 \Delta_{N} \geq$ $N$ ! $-2 \Delta_{N}$, which proves (ii) with a suitable function $f_{h+1}(n)$.

To prove (iii) we assume that $\Phi_{N-1} \leq x \leq \Phi_{N}$. Since $g(N)$ is strictly increasing, $g(N+2 h) \geq g(N+1)+h$. This implies that

$$
\begin{gathered}
x \leq \Phi_{N}=(g(N+1)+h)(g(N+1)-1) ! \\
\leq g(N+2 h)(g(N+1)-1) !<g(N+2 h) !+N+2 h
\end{gathered}
$$

and

$$
x \geq \Phi_{N-1}>g(N) !+h(N-1) ! \geq g(N-1) !+N-1 .
$$

Therefore, we have $A_{i}(N) \leq B_{i}(x) \leq A_{i}(N+2 h)$ for every $1 \leq i \leq h$. Thus we have, $B_{i}(x)=A_{i}(N)+O(1)=(1+o(1)) A_{i}(N)$ as $x \rightarrow \infty$ for every $1 \leq i \leq h$. Now, we have

$$
B_{1}(x) \cdots B_{h}(x)=(1+o(1)) A_{1}(N) \cdots A_{h}(N)=(1+o(1)) N
$$

as $x \rightarrow \infty$. It remains to prove that $B_{h+1}(x)=\frac{x}{N}(1+o(1))$ as $x \rightarrow \infty$. It follows from the definition of $B_{h+1}$ that for $x \geq \Phi_{5}$ we have

$$
\begin{gathered}
B_{h+1}(x)=\Phi_{5}+\sum_{n=6}^{N-1}\left(\frac{\Phi_{n}}{\Delta_{n}}-\frac{g(n) !}{\Delta_{n}}+3\right)+\left\lfloor\frac{x}{\Delta_{N}}-\frac{g(N) !}{\Delta_{N}}+3\right\rfloor \\
=O(N)+\sum_{n=6}^{N-1}\left(\frac{\Phi_{n}}{\Delta_{n}}-\frac{g(n) !}{\Delta_{n}}\right)+\left(\frac{x}{\Delta_{N}}-\frac{g(N) !}{\Delta_{N}}\right) .
\end{gathered}
$$

By $x \geq \Phi_{N-1} \geq N$ !, we have $O(N)=o\left(\frac{x}{N}\right)$ as $x \rightarrow \infty$. It follows from (2) in Lemma 2.1 that $n \geq f_{h}(n)$. Then by the definition of $g(n)$, we have

$$
g(n) \geq g\left(f_{h}(n)\right) \geq n^{2} .
$$

Applying this observation, a straightforward computation shows that

$$
\frac{\Phi_{n}}{\Delta_{n}}-\frac{g(n) !}{\Delta_{n}}=\left(1+O\left(\frac{1}{n^{2}}\right)\right) \cdot \frac{\Phi_{n}}{\Delta_{n}}=\left(1+O\left(\frac{1}{\sqrt{n}}\right)\right) \cdot \frac{g(n+1) !}{n+1} .
$$

Hence,

$$
\sum_{n=6}^{N-1} \frac{\Phi_{n}}{\Delta_{n}}-\frac{g(n) !}{\Delta_{n}}=\sum_{n=6}^{N-1}\left(1+O\left(\frac{1}{\sqrt{n}}\right)\right) \cdot \frac{g(n+1) !}{n+1}
$$


In the next step, we show that

$$
\sum_{n=6}^{N-1}\left(1+O\left(\frac{1}{\sqrt{n}}\right)\right) \cdot \frac{g(n+1) !}{n+1}=(1+o(1)) \cdot \frac{g(N) !}{N}
$$

as $N \rightarrow \infty$. Since $g(m)$ is strictly increasing,

$$
\frac{g(N+1) !}{g(N) !} \geq \frac{(g(N)+1) !}{g(N) !}=g(N)+1 \geq N+1 \geq \frac{N+1}{N},
$$

which implies that $\frac{g(N) !}{N}$ is monotone increasing. By $g(m) \geq m^{2}$, we have

On the other hand,

$$
g(N-1) ! \leq \frac{1}{N^{2}} g(N) !
$$

$$
\frac{g(N-1) !}{N-1} \leq \frac{g(N) ! / N^{2}}{N-1}=O\left(\frac{g(N) !}{N^{3}}\right) .
$$

By using the above observations, we have

$$
\begin{gathered}
\sum_{n=6}^{N-1}\left(1+O\left(\frac{1}{\sqrt{n}}\right)\right) \cdot \frac{g(n+1) !}{n+1}=\sum_{n=7}^{N-1}\left(1+O\left(\frac{1}{\sqrt{n}}\right)\right) \cdot \frac{g(n) !}{n}+\frac{g(N) !}{N}(1+o(1)) \\
=\sum_{n=7}^{N-1} O\left(\frac{g(N-1) !}{N-1}\right)+(1+o(1)) \frac{g(N) !}{N} \\
=O\left(N \frac{g(N) !}{N^{3}}\right)+\frac{g(N) !}{N}(1+o(1))=\frac{g(N) !}{N}(1+o(1))
\end{gathered}
$$

as $x \rightarrow \infty$. It is clear that

$$
\begin{aligned}
\frac{x}{\Delta_{N}}-\frac{g(N) !}{\Delta_{N}} & =\left(1+O\left(\frac{1}{\sqrt{N}}\right)\right)\left(\frac{x-g(N) !}{N}\right) \\
& =(1+o(1)) \frac{x-g(N) !}{N}
\end{aligned}
$$

as $x \rightarrow \infty$. Then it follows that

$B_{h+1}(x)=o\left(\frac{x}{N}\right)+(1+o(1)) \frac{g(N) !}{N}+\frac{x-g(N) !}{N}(1+o(1))=(1+o(1)) \frac{x}{N}$ as $x \rightarrow \infty$, which proves (iii). The proof of Lemma 2.1 is completed.

2.2. Proof of Theorem 1.4. Now, we prove Theorem 1.4 by induction on $h$. We show that there exist infinite sets $A_{1}, \ldots, A_{h} \subset \mathbb{N}$ with the following properties:

(1) $A_{1}+\ldots+A_{h}=\mathbb{N}$,

(2) there exists a monotone increasing arithmetic function $f_{h}(n) \geq 0$ with

$$
\lim _{n \rightarrow \infty} f_{h}(n)=\infty
$$

such that the equation $a_{1}+\ldots+a_{h}=n, a_{i} \in A_{i}$ has a solution with $a_{i} \geq f_{h}(n)$,

(3) $A_{1}(x) \cdots A_{h}(x)=(1+o(1)) x$ as $x \rightarrow \infty$. 
For $h=1$ consider the set of natural numbers and the function $f_{1}(n)=n$, which gives the result. Assume that the statement of Theorem 1.4 holds for $h$. For $h+1$ the result follows from Lemma 2.1. (Actually, for $h=2$ our construction is the same as the construction of Danzer [2]). The proof of Theorem 1.4 is completed.

\section{Proof of Theorem 1.6}

Let $h \geq 2$. We will prove that $\mathcal{A}_{h+1} \subseteq \mathcal{A}_{h}$. Let $A \in \mathcal{A}_{h+1}$. Then there exist $A_{2}, \ldots, A_{h+1} \subseteq \mathbb{N}$ such that $A+A_{2}+\ldots+A_{h+1}=\mathbb{N}$ and $A(x) A_{2}(x) \cdots A_{h+1}(x)=(1+o(1)) x$ as $x \rightarrow \infty$. Let $A_{h}^{*}=A_{h}+A_{h+1}$. It is clear that $A_{h}^{*}(x) \leq A_{h}(x) \cdot A_{h+1}(x)$. Then we have

$$
A+A_{2}+\ldots+A_{h-1}+A_{h}^{*}=\mathbb{N}
$$

and so $A(x) A_{2}(x) \cdots A_{h-1}(x) A_{h}^{*}(x) \geq x+1$. On the other hand,

$$
A(x) A_{2}(x) \cdots A_{h-1}(x) A_{h}^{*}(x) \leq A(x) A_{2}(x) \cdots A_{h+1}(x)=(1+o(1)) x
$$

as $x \rightarrow \infty$, thus we have

$$
A(x) A_{2}(x) \cdots A_{h-1}(x) A_{h}^{*}(x)=(1+o(1)) x
$$

as $x \rightarrow \infty$, which implies that $A \in \mathcal{A}_{h}$. The proof of Theorem 1.6 is completed.

Acknowledgements. This first author was supported by the National Research, Development and Innovation Office NKFIH Grant No. K115288 and K129335. This paper was supported by the János Bolyai Research Scholarship of the Hungarian Academy of Sciences. Supported by the ÚNKP20-5 New National Excellence Program of the Ministry for Innovation and Technology from the source of the National Research, Development and Innovation Fund. The second author was supported by MTA-BME Lendület Arithmetic Combinatorics Research Group. This author was supported by the NKFIH Grants No. K129335. Research supported by the Lendület program of the Hungarian Academy of Sciences (MTA), under grant number LP2019-15/2019.

\section{REFERENCES}

[1] Y.-G. Chen and J.-H. Fang, On a conjecture of Sárközy and Szemerédi, Acta Arith., 169 (2015), 47-58.

[2] L. Danzer, Über eine Frage von G. Hanani aus der additiven Zahlentheorie, J. Reine Angew. Math, 214/215 (1964), 392-394. 
[3] P. Erdős, Some unsolved problems, Michigan Math., J., 4 (1957), 291300.

[4] W. Narkiewicz, Remarks on a conjecture of Hanani in additive number theory, Colloq. Math., 7 (1959/60), 161-165.

[5] I. Z. Ruzsa, An asymptotically exact additive completion, Studia Sci. Math. Hungar., 32 (1996), 51-57.

[6] I. Z. Ruzsa, Additive completion of lacunary sequences, Combinatorica, 21 (2001), 279-291.

[7] A. Sárközy and E. Szemerédi, On a problem in additive number theory, Acta Math. Hungar., 64 (1994), 237-245.

Institute of Mathematics, Budapest University of Technology and EcoNOMics, Egry József utca 1, 1111 Budapest, Hungary

Email address: ksandor@math.bme.hu

Institute of Mathematics, Budapest University of Technology and EcoNOMics, Egry József utca 1, 1111 Budapest, Hungary

Email address: csandor@math.bme.hu 